\title{
Engineering Creativity: The Influence of General Knowledge and Thinking Heuristics
}

\author{
louri Belski, Anne Skiadopoulos, Guillermo Aranda- \\ Mena, Gaetano Cascini, and Davide Russo
}

\section{Introduction}

\subsection{The Need for Creative Solutions Beyond Profession}

Rapid change in technology, growing availability of new materials as well as development of highly accurate models that underpin computer-based

I. Belski $(\bowtie) \bullet G$. Aranda-Mena

Royal Melbourne Institute of Technology, Melbourne, VIC, Australia e-mail: iouri.belski@rmit.edu.au
A. Skiadopoulos
La Trobe University, Bundoora, VIC, Australia
G. Cascini
Polytechnic University of Milan, Milan, Italy
D. Russo

Department of Management, Information and Production Engineering,

University of Bergamo, Bergamo, Italy

e-mail: davide.russo@unibg.it 
simulators have resulted in shrinking lifetime of products and services created by engineers. These changes in resources available to engineering designers brought restrictions on product development time and raised a demand for creativity of engineering solutions. In order to win in this rapidly changing world, engineering companies need to offer novel products that are capable of outperforming those of their competitors. Moreover, these novel products need to have reasonably long life spans in order for companies to make profits and to prevent themselves from being outcompeted by new products of others. These constraints require engineering designers to gain skills in choosing winning designs from numerous suitable proposals that are based not only on diverse external appearances but also on different principles of operations.

Growing availability of advanced manufacturing technologies enables engineers to develop the same design functionality in more than one way (e.g. mechanically, chemically, electro-magnetically, deploying nanotechnology, etc.). Therefore, in order to succeed in today's rapidly changing world, engineers need to be abreast of novel technologies. This will help them to continuously deliver innovative products by choosing the most suitable technologies and principles of operation that suit the required product functionality.

Development of silicon wafer cleaning methods illustrates the changes in cleaning technologies that are based on different principles of operation. Over the last 60 years, cleaning methods evolved from traditional mechanical and chemical cleaning to vapor-phase cleaning, plasma stripping and cleaning as well as cryogenic aerosol/supercritical fluid cleaning (Kern 2008). Furthermore, the evolution of silicon wafer cleaning technologies on novel principles of operation is still continuing with new cleaning methods developed continuously (e.g. foam/bubble cleaning, laser cleaning, nanoprobe cleaning) (Reinhardt 2008).

In essence, changes in technology resources have strengthened the need for engineering designers to propose divergent ides for product implementation. Engineers of the twenty-first century need skills in choosing the most 'progressive' and 'winning' designs for development and fabrication. More and more often, these choices can be found outside of a knowledge base of a single engineering profession. 


\subsection{Definition of Engineering Creativity}

Recent studies advocated that creativity is domain specific (Baer 2015). More and more scholars agree that being creative in one domain does not make an individual creative in another knowledge domain (Weisberg 2006; Baer 2012). This view is further supported by negligible transfer of the creativity training gains from one knowledge area to another (Baer 2016). Therefore this chapter will specifically consider engineering creativity and use the definition of engineering creativity developed by Belski (2017):

Engineering creativity is the ability to generate novel solution ideas for openended problems, ideas that are not obvious to experts in a particular engineering discipline and that are considered by them as potentially useful. (Belski 2017, p. 327)

This definition is based on analysis of legal criteria of patentability and patent authorship. It implies that the knowledge outside of the discipline is essential for engineering creativity. In order for an idea to not be obvious to experts from a particular discipline, it needs to be somewhat unusual for this discipline. In other words, to be considered as creative in engineering an idea has to use information that is not well known to professionals from this discipline (i.e. located 'outside' of their discipline knowledge).

\subsection{Sources of Engineering Creativity}

In her pioneering work on creativity that is still considered as appropriately describing creative performance, Amabile (1983) suggested that creative performance depends on three main components: domain-relevant skills, (2) creativity-relevant skills and (3) task motivation. Domain-relevant skills include domain knowledge and domainrelevant 'talent' and depend on innate cognitive abilities as well as on formal and informal education of an individual. Creativity-relevant skills comprise appropriate cognitive style and knowledge of ideation heuristics 
and depend on training and experience. Task motivation incorporates problem solver's attitudes towards the task and depends on his or her intrinsic motivation (Amabile 1983).

It appears that perpetual increase in supply of resources that engineering designers can use, which has been occurring over the last 20-30 years, advocates for the extension of the above-mentioned creativity components proposed by Amabile. More and more evidence that originated from industrial corporations demonstrate that the knowledge outside of profession is becoming essential for engineering creativity.

This importance of general knowledge, for example, is supported by the findings made by Belski, Adunka and Mayer (2016a), who reported on the outcomes of surveying 46 engineering experts from the most innovative world corporations. Belski et al. established that, although discipline knowledge, years of practice (i.e. experience) and proficiency with creativity techniques are still considered by the experts important for achieving creative designs, general knowledge outside of their profession has become more essential for creative performance. The paired-samples t-test of the means of survey responses showed statistically significant differences between the need of general knowledge versus discipline knowledge $(t=4.3, p<0.001)$ as well as between the need of general knowledge versus years of practice $(t=3.8, p<0.001)$ for creative engineering work (Belski et al. 2016a). Such positive view of engineering experts on the role of general knowledge in creativity is well expressed by the above-mentioned definition of engineering creativity. In order for an idea to be not obvious to experts in a particular engineering domain, it needs to integrate knowledge outside of this domain. Otherwise, the idea will be 'obvious' to the domain experts.

Accordingly, in order to adequately model creative performance of engineers of the twenty-first century, knowledge from outside of the profession needs to be incorporated as an additional module into the Amabile's components.

\subsection{Expert Schemas Versus Memory Search by Novices}

The difference in problem-solving strategies between experts and novices has been extensively studied over the last 60 years (e.g. Gick 1986; Simon 1996; Harlim and Belski 2013, 2017; Cross 2004; Weisberg 2006). It has 
been concluded that one of the key differences in problem solving between experts and novices is associated with expert knowledge schemas. Over the years of practice, experts develop effective schemas that arm them with excellent task-recognition skills and allow them to propose sound solutions in their professional domain quickly (Belski and Belski 2013; Simon 1996). Novices, on the other hand, have not had sufficient practical experience to build effective knowledge schemas and, therefore, require searching a problem space in order to find solutions (Belski and Belski 2008; Gick 1986). Although expert knowledge schemas have been considered as advantageous in problem solving, it was posited that they may hinder experts' creativity (Belski and Belski 2013). Moreover, it was suggested that one of the effective ways for enhancing creativity of experts lies in engaging them in deliberate search of their knowledge repository for solution ideas that lie beyond their area of expertise (Belski and Belski 2013).

The suggestion that deliberate search of own general knowledge can bring more novel ideas to experts was confirmed by the study of Dobrusskin, Belski, and Belski (2014). They surveyed a team of 13 experts from Philips who were involved in developing solutions for a technical problem that had been thoroughly protected by intellectual property by other companies. In order to search their knowledge, these experts used the Theory of Inventive Problem Solving (TRIZ) heuristic of systematised Substance-Field Analysis (Su-Field) (Belski 2007). Although prior to utilisation of Su-Field they spent two weeks on situation and function analyses and were involved in industry scouting, the team did not find many suitable solutions. Su-Field, together with the eight fields of Mechanical, Acoustic, Thermal, Chemical, Electric, Magnetic, Intermolecular and Biological (MATCEMIB) that it uses for idea search, was much more helpful for generating novel ideas. Most of the survey participants believed that 'the use of the Su-Field procedure generated ideas that would have been overlooked otherwise' (p. 126). Furthermore, the survey participant agreed that 'the eight fields of MATCEMIB have helped [them] to thoroughly search [their] knowledge for solution ideas' (p. 126).

\subsection{Enhanced Idea Generation with Su-Field}

Recent studies reported on the positive influence of systematised Su-Field on students' ability to generate diverse ideas for a knowledge-rich, open-ended 
engineering problem (how to clean lime deposits from inside water pipes) (Belski et al. 2014, 2015, 2016b). It was discovered that the first-year students from universities in Australia, Czech Republic, Finland and Russia who were simply exposed to the names of the eight fields of MATCEMIB during idea generation produced at least two times more ideas than the students from a control group that were not shown any prompts. Also, students that were exposed to the names of the eight fields of MATCEMIB proposed solution ideas that covered a significantly broader range of engineering principles of operation (Belski et al. 2015). The experiment conducted with university students in Germany, which involved undergraduate and postgraduate engineering students, supported the findings of the above-mentioned experiments with the first-year students. It also recorded a boost in both a number of distinct ideas and breadth of these ideas for the students who were shown the names of the eight fields of MATCEMIB (Belski et al. 2016b).

\subsection{General Knowledge and Differences in Performance}

The results of the above-mentioned idea-generation experiments showed significant differences in the numbers and the breadth of ideas generated by the control groups of students from all five countries. Table 1 depicts the information on the composition of the control groups, semester of study at university, an average number (mean) of independent ideas generated by students of a particular group and the breadth of these ideas (Belski et al. 2015, 2016b).

Table 1 A number (mean) and breadth of distinct ideas generated by students from control groups

\begin{tabular}{llll}
\hline Country & Students & Breadth & Mean \\
\hline Australia & $21(\mathrm{~s} 1)$ & 2.05 & 2.00 \\
Czech Republic & $18(\mathrm{~s} 1)$ & 2.53 & 3.56 \\
Russia & $21(\mathrm{~s} 1)$ & 2.57 & 4.32 \\
Finland & $8(\mathrm{~s} 1)$ & 2.75 & 5.81 \\
Germany & $37(\mathrm{~s} 3)$ & 2.30 & 3.90 \\
\hline
\end{tabular}


The breadth of ideas was calculated as a sum of eight terms, each equal to a fraction of students from the control group that proposed ideas that were assigned by the assessors to each field of MATCEMIB (Belski et al. 2015). For example, the following is the spread of the ideas proposed by the students from Australia: 95\% of students proposed Mechanical ideas; 5\% - Acoustic; 14\% - Thermal; 86\% - Chemical; 0\% - Electric; 0\% Magnetic; 0\% - Intermolecular; 5\% - Biological. Therefore, the breadth of ideas proposed by the control group from Australia was equal to:

$$
\text { Breadth }=0.95+0.05+0.14+0.86+0+0+0+0.05=2.05
$$

Belski and Belski (2016) analysed the results of students from Australia, Czech Republic, Russian Federation and Finland presented in Table 1 and concluded that the difference in the numbers and the breadth of ideas generated by students from different countries can be explained by dissimilarities in the following: (a) the depth of their knowledge in science that has been acquired during secondary school study and (b) entry prerequisites to degrees at the universities that participated in the study. Students from countries with better educational systems, who were required to demonstrate their sound knowledge in science prior to entering engineering study at university, proposed more independent ideas that also covered a wider set of principles of operation (Belski and Belski 2016).

The conclusion that science knowledge influences the breadth and the number of the ideas proposed by individual students has recently been supported by a study by Buskes and Belski (2017) that repeated the above-mentioned lime deposit cleaning experiment with the students from the University of Melbourne. It was discovered that both the breadth and the number of the ideas generated by a student from the control group at the University of Melbourne were moderately and statistically significantly correlated with the number of science subjects the student completed at secondary school. Moreover, the number of science subjects studied by the student explained $17 \%$ variation in the number and the breadth of the ideas proposed (Buskes and Belski 2017). These results further support the inclusion of the component of 'general knowledge 
outside the professional domain' into the creativity model developed by Amabile.

Almost all students from Australia, Czech Republic, Russian Federation and Finland entered university directly from secondary school. Therefore, they have not acquired substantial discipline knowledge and did not have enough practical experience for the expert schemas to form in their engineering disciplines. This means that whilst generating ideas for cleaning pipes of lime deposit they searched their database of general knowledge in a way expected from novices. Table 1 (Breadth) and the Breadth formula (1) indicate that this search for solutions was not very efficient. Most of the ideas proposed by the students from all control groups recommended solutions based on two principles of operation: either cleaning the lime deposit from pipes mechanically or by using some chemical substance to remove it.

Students from the German control group were in their second year of study. The first year of engineering study at university is usually devoted to introduction to the profession and to expansion of general knowledge. Therefore, it was anticipated that these students gained more knowledge outside of their profession than the students from the other four control groups who had just entered university did. Hence, the students from Germany were expected to perform better than the students that had just entered universities. As shown in Table 1, this did not occur. Moreover, although the results of the experimental groups from Germany that combined students of different study years, which are discussed in Belski et al. (2016b), were aligned with the results for the experimental groups from the other four countries as presented in Belski et al. (2015), the influence of general knowledge versus discipline knowledge on idea generation was not fully clear. In order to establish the influence of experience and knowledge on creative performance more accurately, it was necessary to analyse idea-generation performance of users with practical experience and education that significantly exceeded that of the first-year students from universities in Australia, Czech Republic, Finland and Russia.

This chapter presents the results of the study that repeated the experiments conducted in the five countries at two universities in Italy. This time, all 64 students that participated in the study were enrolled in the engineering master's programs. The study planned to establish correctness 
of two hypotheses that have been discussed by Belski et al. (2016a): (1) general knowledge is more important than discipline knowledge for attaining creative solution ideas in engineering and (2) engaging a user in searching the person's knowledge repository by prompting a user with the eight fields of MATCEMIB of Su-Field accelerates idea generation more effectively than additional discipline knowledge and years of practical experience.

\section{Methodology}

Sixty-four master's students from the University of Bergamo and the Polytechnic University of Milan, who were in their seventh or eighth semester of university, participated in this study that repeated the experiment conducted by Belski et al. (2014). The following is a short record of activities that the participants were involved in.

At each university, students were divided into four tutorial groups: one control and three experimental. All students were given 16 minutes of tutorial time to individually generate as many ideas as possible for the same problem (to remove the lime build-up in water pipes). Initially, the same PowerPoint slide that contained the problem statement translated into Italian and a photo of a cross-section of a pipe, half of which was covered with lime deposit, was presented to the students for two minutes by their tutors. Figure 1a depicts the English version of the problem statement that was presented to students from all groups. After two minutes of problem introduction that covered only the information presented in Fig. 1a, all students were asked to work individually and to record as many ideas to clean the lime build-up from the pipes as possible (ideas were recorded by students in Italian). The form to record ideas was distributed to the students just before the problem was presented. The form was the same for the students of all four groups. It was a copy of the Australian form that was translated into Italian.

Students from the control group were not influenced by any ideation methodology. After two minutes of problem introduction, they were allowed to think of solution ideas and to record them for 16 minutes. The slide shown in Fig. 1a was presented to the students from the control group for the whole duration of the idea-generation session. 
Calcium carbonate, or lime, is a hard deposit found in kettles, the inner surface of pipes and other surfaces.

How to Remove the Lime Build Up in Pipes?

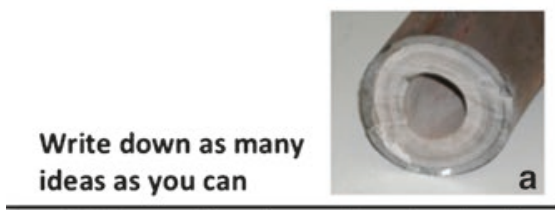

Calcium carbonate, or lime, is a hard deposit found in kettles, the inner surface of pipes and other surfaces.

How to Remove the Lime Build Up in Pipes?

\section{Biological}

Write down as many ideas as you can

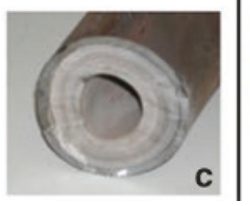

Calcium carbonate, or lime, is a hard deposit found in kettles, the inner surface of pipes and other surfaces.

How to Remove the Lime Build Up in Pipes?

\section{Lotus eater}

Write down as many ideas as you can

Calcium carbonate, or lime, is a hard deposit found in kettles, the inner surface of pipes and other surfaces. How to Remove the Lime Build Up in Biological Pipes?

Microbes, bacteria, living organisms, plants, fungi, cells, enzymes

Write down as many ideas as you can

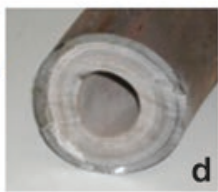

Fig. 1 The English version of the PowerPoint slides presented to students in Italian: (a) task introductory and the Control Group; (b) Random Word group; (c) MATCEMIB group; (d) MATCEMIB+ group. (Belski et al. 2015)

After two minutes of problem presentation, students from the experimental groups were told that during their idea-generation session they would be shown some words. No explanation of what these words were and what to do with them were given. Students from the Random Word groups were offered the Italian translation of the eight random words that were used in all other experiments. Students from the MATCEMIB group were offered the translations of the names of the eight fields of MATCEMIB in Italian. The MATCEMIB+ group students were shown the names of the eight fields (in big font) as well as some words (in small font) that illustrated the interactions of the particular field (e.g. friction, direct contact, collision, wind, etc. for the Mechanical field). The name of each field as well as each random word was shown to the students from the experimental groups for two minutes. Every two minutes a tutor changed the word on the screen and read the new word aloud. When a 
tutor of the MATCEMIB+ group changed slides every two minutes, he read aloud only the name of the field of MATCEMIB that was displayed, but did not read the words that illustrated field interactions that were displayed together with the field's name. Altogether, the students from all groups were generating and recording ideas for 16 minutes.

Figure 1 depicts the English version of one of the eight PowerPoint slides that were shown to the Italian students: Fig. 1a - the Control groups; Fig. 1b - the Random Word groups; Fig. 1c - the MATCEMIB groups; Fig. 1d - the MATCEMIB+ groups.

\section{$3 \quad$ Results}

\subsection{Italian Study Only}

Two independent assessors that used the same criteria as assessors from all other counties evaluated student ideas at each university. These criteria were developed for the original Australian study (Belski et al. 2014). Among other items, assessors counted the number of distinct (independent) ideas proposed by each student. In order to judge how broad or 'divergent' these independent ideas were, each idea was assigned to a field of MATCEMIB that most corresponded to the proposed principle of operation. The inter-rater reliability of assessment by independent assessors was evaluated for each university separately with SPSS software by establishing the Cronbach's Alpha for the number of independent ideas proposed by each individual student. Cronbach's Alphas for both the University of Bergamo and the Polytechnic University of Milan were very close to 0.9 . The Cronbach's Alpha coefficient of 0.9 indicates excellent internal consistency. Thus, the assessment of students from both universities was evaluated as very reliable. As expected, due to similar students' backgrounds, the results of students from the same groups at both universities were very similar. Therefore, for further analysis the number of independent ideas proposed by each individual student made by the assessors was averaged and the results of students from both universities were combined. 
Table 2 A number (Mean) and the Breadth of distinct ideas generated by students from Italy

\begin{tabular}{llll}
\hline Group & Students & Breadth & Mean \\
\hline Control & $16(s 7,8)$ & 2.8 & 4.4 \\
Random Word & $15(s 7,8)$ & 3.0 & 6.5 \\
MATCEMIB & $18(s 7,8)$ & 4.7 & 6.4 \\
MATCEMIB+ & $15(s 7,8)$ & 6.1 & 8.1 \\
\hline
\end{tabular}

Table 2 presents the results for the average number of independent ideas proposed by the Italian students in each group (mean) and the breadth of these ideas. It also contains information on the group sizes and their study semester.

The number of distinct solution ideas proposed by students was distributed normally in all groups, therefore one-way ANOVA with post-hoc Bonferrony tests were conducted. ANOVA showed significant differences between the groups $(F=8.545, p<0.001)$. Bonferrony tests revealed statistically significant differences between the control group and each of the three experimental groups. Differences in the numbers of independent ideas between the experimental groups were not statistically significant.

The distribution of breath of ideas was not normal in some of the groups; therefore, the Kruskal-Wallis test of independent samples was conducted. It showed statistically significant difference in breadth between the groups $(p<0.001)$. The Matt-Whitney $U$ tests were conducted to establish statistical differences in breadth of ideas between the groups. They showed statistically significant difference in breadth of ideas between the control group and the MATCEMIB group $(Z=-3.896, p<0.001)$ as well as the MATCEMIB+ group $(Z=-4.068, p<0.001)$. The tests also revealed statistically significant difference in breadth of ideas between the Random Word group and the MATCEMIB group $(Z=-3.625$, $p<0.001)$ as well as the MATCEMIB $+\operatorname{group}(Z=-3.918, p<0.001)$. Differences between all other groups were not statistically significant.

\subsection{Control Groups: Italians and Students from Other Counties}

The number of solution ideas in all control groups was distributed normally, therefore one-way ANOVA with post-hoc Bonferrony tests were 
conducted to establish differences in performance of the control groups of the first-year students with that of the master's students. ANOVA displayed significant statistical differences between the control groups for the number of ideas $(F=7.050, p<0.001)$. Bonferrony tests showed that the Italian control group statistically significantly outperformed only the control group from Australia. Differences in the numbers of independent ideas between the control groups from Italy and the other four countries were not statistically significant.

The distribution of breath of ideas was not normal in some control groups; therefore, the Kruskal-Wallis test of independent samples was conducted. The test showed that the distribution of the ideas' breadth between the control groups was not statistically significant.

\section{Discussion}

\subsection{General Knowledge Versus Discipline Knowledge}

Comparison of performance of the control group from Italy with that from the other five countries only partly supported the first hypothesis (general knowledge is more important than discipline knowledge for attaining creative solution ideas). This conclusion was made on the basis of the following analysis.

Secondary schooling of students from Italy, which is a part of the European Union, as well as university entry requirements for engineering degrees in Italy, had more similarity to that of Czech Republic, Finland, Germany and even the Russian Federation than to Australia. Therefore, likewise to the conclusions of Belski and Belski (2016), statistically significant difference in the number of ideas proposed by the control groups from Italy and Australia can be explained by significant differences in science (general) knowledge of students from these two groups. This explanation supports the first hypothesis.

The absence of statistically significant differences in the number and breadth of solution ideas proposed by the students from the control groups from Czech Republic, Finland, Russian Federation, Germany and 
Italy suggests that extension of discipline knowledge and practical experience that the Italian students attained over at least four years of extra study caused minimal influence on their creative performance. Assuming that the Italian students have not gained much general knowledge over the four years, this conclusion also supports the first hypothesis.

Indeed, significant expansion of general knowledge by the Italian students seems very unlikely. Engineering curricula are overloaded with discipline-related subjects and are focused on specialisation of students in their professions. Only very few subjects taught to engineering students over the three years of bachelor's degree programs in the European Union are devoted to expansion of their general knowledge. This means that the Italian students had significantly extended professional knowledge to that of the students from Czech Republic, Finland, Germany and the Russian Federation. At the same time, the gain in general knowledge by the Italian students over the additional four years of university study as well as due to extra practical experience might have been minimal and insufficient for enhancing their creative performance.

Alternatively, it is possible that over the four years of study students from Italy also substantially expanded their general knowledge (that is outside of their profession). Hence, if the first hypothesis is true, and additional general knowledge enhances creativity, Italian control group students were expected to outperform students from the control groups from all other countries, not only the students from Australia. Actually, Italian students performed somewhat better than students from the control groups of similar size. They did slightly better than their counterparts from Germany, Czech Republic and the Russian Federation in both the number of ideas ( 4.40 versus 3.90; 3.56 and 4.32 respectively) and the breadth of ideas $(2.80$ versus $2.30 ; 2.53$ and 2.57$)$. The fact that the difference in performance of Italian students was not statistically significantly higher than that of their peers from Germany, Czech Republic and the Russian Federation may imply that the gain in general knowledge by Italian students over the additional four years of study was not big enough to result in statistical significant difference in idea-generation performance.

Still, the assumption that Italian students gained substantial general knowledge over the four years cannot be ruled out. If it is the case, poor 
improvement in creative performance by the Italian control group compared to their first-year counterparts could be explained by reframing the interpretation of the opinions of experts surveyed by Belski, Adunka, and Mayer (2016a) on superiority of general knowledge over discipline knowledge for attaining creative solutions in engineering. The study of Buskes and Belski (2017) established that the variation of the number and the breadth of generated ideas that is explained by differences in science knowledge is quite moderate (17\%). This means that extra general knowledge may not effectively facilitate improvement in creative performance on its own. Such conjecture does not contradict with the conclusions of Belski and Belski (2016) and Buskes and Belski (2017) on the influence of extensive prior knowledge in science on creative performance of engineering students. It just advocates reconsidering modelling the way general knowledge influences creativity. Most likely, students from the Italian control group were unable to use their additional general knowledge effectively, so their performance did not significantly differ statistically from that of the first-year students from the control groups.

It can be posited that extra general knowledge does not enhance creative performance on its own unless it has been transformed into appropriate problem-solving schemas. And in order for the general knowledge that has not been 'schematised' to lift creativity, it requires a 'catalyst' that helps a user to utilise his or her general knowledge effectively. The need for a catalyst is also supported by the opinions of the experts from the Belski et al. (2016a) study. The study engaged engineering experts from the most innovative world companies, who had good knowledge of ideation techniques, and also practiced them regularly. It is possible that regular practice in creativity techniques helped the experts to utilise their general knowledge and generate novel solution ideas effectively. This might explain why the experts' agreement with the statement 'creativity techniques that I have learnt over the years have significantly improved my ability to solve engineering problems creatively' $(7.74 / 10)$ was second highest after the importance of general knowledge (8.41/10) and exceeded in value the discipline knowledge $(7.00 / 10)$ and practical experience (7.21/10) (Belski et al. 2016a).

Thus, in order for the first hypothesis to be fully supported by the experimental data that is available so far, it needs to be reformulated as: 
'general knowledge is more essential for creative performance than discipline knowledge if a practitioner is capable of searching his/her knowledge data base for general knowledge effectively'.

\subsection{Idea Generation with Su-Field: Influence of MATCEMIB}

The outcomes of the experiment conducted in Italy with the master's degree students were similar to the outcomes of the experiments that engaged the first-year bachelor's degree students from four other countries (Belski et al. 2015). The influence of the eight fields of MATCEMIB on both the number of ideas generated and on the breadth of these ideas had been fully replicated. Italian students from the MATCEMIB and the MATCEMIB+ groups proposed statistically significantly more ideas than their counterparts from the control group. The differences in breadth of solution ideas between each of the MATCEMIB groups and the control group were also statistically significant.

In essence, the results of idea generation of students from the Italian control group, those who have completed their bachelor's degrees, those may have had practical experience in industry and those who have returned back to university to get master's degrees, matched that of the control groups of recent school leavers but was well below the performance of the recent school leavers from the MATCEMIB and MATCEMIB+ groups from the other five countries. This means that the additional knowledge that the Italian students gained over four years of studying engineering did not make as significant a positive influence on their ability to utilise their knowledge as the prompts of the eight fields of MATCEMIB that were shown to the first-year students from Australia, Czech Republic, Finland and the Russian Federation. It appears that the names of the fields of MATCEMIB acted as the catalyst. They engaged students in searching their knowledge repositories and accelerated idea generation much more effectively than significant additional discipline knowledge and years of practical experience the Italian students had gained over at least four years. These results support the second hypothesis (engaging a user in searching the person's knowledge repository by 
prompting a user with the eight fields of MATCEMIB of Su-Field accelerates idea generation more effectively than additional discipline knowledge and years of practical experience).

\section{Conclusions}

The results of this study support the need to incorporate the component of 'general knowledge' into the model of creativity developed by Amabile (1983) in order to make this model suitable for the engineering profession of the twenty-first century. Due to a rapid change in technologies and growing availability of new materials, expert engineers can achieve patentable solutions when their knowledge spans beyond their traditional domain knowledge. Moreover, it seems that in order for engineering experts to utilise this general knowledge effectively, they need to apply sound ideation heuristics, like that of the eight fields of MATCEMIB.

\section{References}

Amabile, T. M. (1983). The social psychology of creativity: A componential conceptualization. Journal of Pelsonality and Social Psychology, 45(2), 357-376. Baer, J. (2012). Domain specificity and the limits of creativity theory. The Journal of Creative Behavior, 46(1), 16-29. https://doi.org/10.1002/jocb.002. Baer, J. (2015). The importance of domain-specific expertise in creativity. Roeper Review, 37(3), 165.

Baer, J. (2016). Content matters: Why nurturing creativity is so different in different domains. In R. A. Beghetto \& B. Sriraman (Eds.), Creative contradictions in education (pp. 129-142). Switzerland: Springer International Publishing.

Belski, I. (2007). Improve your thinking: Substance-field analysis. Melbourne: TRIZ4U.

Belski, I. (2017). Engineering creativity - How to measure it? In N. Huda, D. Inglis, N. Tse, \& G. Town (Eds.), Proceedings of the 28th annual conference of the Australasian Association for Engineering Education (AAEE 2017) (pp. 321-328). Sydney: School of Engineering, Macquarie University.

Belski, I., \& Belski, I. (2008). Cognitive foundations of TRIZ problem-solving tools. In T. Vaneker (Ed.), Proceedings of the TRIZ-future conference 2008 (pp. 95-102). Enschede: University of Twente. 
Belski, I., \& Belski, I. (2013). Application of TRIZ in improving the creativity of engineering experts. In A. Aoussat, D. Cavallucci, M. Trela, \& J. Duflou (Eds.), Proceedings of TRIZ future conference 2013 (pp. 67-72). Paris: Arts Et Metiers ParisTech.

Belski, I., \& Belski, R. (2016). Influence of prior knowledge on students' performance in idea generation: Reflection on university entry requirements. In S. T. Smith, Y. Y. Lim, A. Bahadori, N. Lake, R. V. Pagilla, A. Rose, \& K. Doust (Eds.), Proceedings of the 27th annual conference of the Australasian association for engineering education - AAEE2016 (pp. 1-9). Lismore: Southern Cross University.

Belski, I., Hourani, A., Valentine, A., \& Belski, A. (2014). Can simple ideation techniques enhance idea generation? In A. Bainbridge-Smith, Z. T. Qi, \& G. S. Gupta (Eds.), Proceedings of the 25th annual conference of the Australasian association for engineering education (pp. 1C, 1-9). Wellington: School of Engineering \& Advanced Technology, Massey University.

Belski, I., Belski, A., Berdonosov, V., Busov, B., Bartlova, M., Malashevskaya, E., et al. (2015). Can simple ideation techniques influence idea generation: Comparing results from Australia, Czech Republic, Finland and Russian Federation. In A. Oo, A. Patel, T. Hilditch, \& S. Chandran (Eds.), Proceedings of the 26th annual conference of the Australasian association for engineering education - AAEE2015 (pp. 474-483). Geelong: School of Engineering, Deakin University, Victoria, Australia.

Belski, I., Adunka, R., \& Mayer, O. (2016a). Educating a creative engineer: Learning from engineering professionals. Procedia CIRP, 39, 79-84. https:// doi.org/10.1016/j.procir.2016.01.169.

Belski, I., Livotov, P., \& Mayer, O. (2016b). Eight fields of MATCEMIB help students to generate more ideas. Procedia CIRP, 39, 85-90. https://doi. org/10.1016/j.procir.2016.01.170.

Buskes, G., \& Belski, I. (2017). Prior knowledge and student performance in idea generation. In N. Huda, D. Inglis, N. Tse, \& G. Town (Eds.), Proceedings of the 28th annual conference of the Australasian association for engineering education (AAEE 2017) (pp. 354-361). Sydney: School of Engineering, Macquarie University.

Cross, N. (2004). Expertise in design: An overview. Design Studies, 25(5), 427-441.

Dobrusskin, C., Belski, A., \& Belski, I. (2014). On the effectiveness of systematized substance-field analysis for idea generation. In C. Tucci, T. Vaneker, \& T. Nagel (Eds.), Proceedings of the TRIZ future conference: Global innovation convention (TFC 2014) (pp. 123-127). Freiburg: The European TRIZ Association. 
Gick, M. L. (1986). Problem-solving strategies. Educational Psychologist, 21(1/2), 99-120.

Harlim, J., \& Belski, I. (2013). Long-term innovative problem solving skills: Redefining problem solving. International Journal of Engineering Education, 29(2), 280-290.

Harlim, J., \& Belski, I. (2017). Stages of engineering problem solving: Learning from the experts. In N. Huda, D. Inglis, N. Tse, \& G. Town (Eds.), Proceedings of the 28th annual conference of the Australasian association for engineering education (AAEE 2017) (pp. 295-302). Sydney: School of Engineering, Macquarie University.

Kern, W. (2008). 1 - Overview and evolution of silicon wafer cleaning technology. In Handbook of silicon wafer cleaning technology (2nd ed., pp. 3-92). Norwich: William Andrew Publishing.

Reinhardt, K. A. (2008). 11 - New cleaning and surface conditioning techniques and technologies. In Handbook of silicon wafer cleaning technology (2nd ed., pp. 661-688). Norwich: William Andrew Publishing.

Simon, H. A. (1996). The sciences of the artificial (3rd ed.). Cambridge, MA: MIT.

Weisberg, R. W. (2006). Creativity: Understanding innovation in problem solving, science, invention, and the arts. Hoboken: Wiley. 